\title{
A new automated procedure of modal identification in operational conditions
}

\author{
Gabriele Marrongelli, ${ }^{1, *}$, and Carmelo Gentile ${ }^{1}$ \\ ${ }^{1}$ Politecnico di Milano, ABC Department, Milan, Italy
}

\begin{abstract}
Structural Health Monitoring (SHM) strategies are aimed at the assessment of structural performance, using data acquired by sensing systems. Among the different available approaches, vibration-based methods - involving the automation of the modal parameter estimation (MPE) and modal tracking (MT) procedures - are receiving increasing attention. In the context of vibration-based monitoring, this paper presents an automated procedure of modal identification in operational conditions. The presented algorithms can be used to effectively manage the results obtained by any parametric identification method that involves the construction and the interpretation of stabilization diagrams. The implemented approach introduces improvements related to both the MPE and the MT tasks. The MPE procedure consists of three key steps aimed at: (1) filtering a high number of spurious poles in the stabilization diagram; (2) clustering the remaining poles that share same characteristics in term of modal parameters; (3) improving the accuracy of the modal parameter estimates. In the MT procedure the use of a simple statistical approach to define adaptive thresholds together with continuously updated dynamic reference list guarantee an efficient tracking of the most representative structural modes. The advantages obtained through the proposed procedures are exemplified using data continuously collected on the historic masonry tower of San Gottardo in Corte, located in the centre of Milan, Italy. In addition, the ability of the automated algorithms to identify contributions inherent to different vibration modes, even if they are characterized by closely-spaced frequencies and a low discriminant between mode shapes, will be described in details.
\end{abstract}

\section{Introduction}

The Stochastic Subspace Identification (SSI) methods are particularly suitable for modal parameters estimation of full-scale structures. SSI methods [1] identify a stochastic statespace model from the measured outputs and the extraction of modal parameters is carried out through the use of state-space models with increasing model order. Values and associated uncertainty are subsequently obtained using the well-known graphical tool called stabilization diagram. The key idea of the stabilization diagram relies on the fact that the poles associated to physical modes, are conceivably identified when consistent modal

\footnotetext{
*Corresponding author: mailto:gabriele.marrongelli@polimi.it
} 
properties are obtained for models of different order, as well as for different time-lag [2]. On the other hand, overestimating the model using a high order implies the appearance of spurious modes associated to the noise content of the signals. These modes do not have physical meaning and should be detected and removed from the diagram making it cleaner for easy detection of the structural dynamic properties. Within the context of automated modal identification, it is necessary to distinguish between two different processes: (i) modal parameter estimation (MPE) and (ii) modal tracking (MT). The former consists of the automated estimation of the modal parameters from a single data-set (collected in a single test or during a permanent monitoring), whereas the latter compares the results coming from the repeated application of MPE to continuously collected data-sets with the objective of tracking the evolution in time of the modal parameters.

\section{Automated modal parameters estimation}

As previously stated, the main objective of an automated MPE procedure is to perform a robust identification of the modal parameters with a low uncertainty value using only the structural response in operation conditions. The MPE procedure herein presented is based on previous contributions available in the literatures [3-5] and consists of the sequential application of three steps: (i) removing a high number of spurious modes; (ii) automatically estimating the modal parameters through a clustering procedure, (iii) improving the accuracy of the modal parameter estimates.

\subsection{Pre-filtering}

The first step, or pre-filtering is aimed at detecting and removing the spurious poles from the stabilization diagram. This task is performed through three single mode criteria based on pre-selected threshold values for damping and mode shape complexity, with the complexity being defined through the Modal Phase Collinearity (MPC) and Mean Phase Deviation (MPD). In fact, in normal operating conditions the behaviour of a structure is strictly stable and the structure is lightly damped: for this reason highly damped modes and high complexity modes that exceed such thresholds are considered not realistic or associated to the noise content of the signal then removed from the diagram [4].

\subsection{Clustering}

The subsequent step consists of a clustering procedure with the objective of grouping all poles that sharing the same characteristics in terms of modal parameters. In fact, once most of the spurious poles have been removed through the pre-filtering check, the most popular way to group stable poles into clusters is to measure the distance of different poles in term of natural frequency and mode shape (using MAC index), using the metric initially proposed by Magalhaes et al. [3] and recently modified by Cabboi et al. [5], so that a reference pole is introduced to select poles that represent the same mode. The distance between the reference pole and "candidate pole" is calculated using the following metric:

$$
d_{i, r e f-j}^{C}=\frac{\left|f_{i, r e f}-f_{i, j}\right|}{f_{i, r e f}}+1-\frac{\left|\phi_{i, r e f}^{H} * \phi_{i, j}\right|^{2}}{\left(\phi_{i, r e f}^{H} * \phi_{i, r e f}\right)\left(\phi_{i, j}^{H} * \phi_{i, j}\right)}
$$

where $f_{i, r e f}$ and $\phi_{i, \text { ref }}$ represents the mean frequency and mean mode shape of the reference pole, whereas $f_{i, j}$ and $\phi_{i, j}$ are the modal parameters corresponding to $j$-th current pole at $i$-th 
model order. A distance threshold was used to group estimates with similar characteristics avoiding incorrect association, it was fix equal to 0.03 . When all poles have been grouped into the clusters, the clusters with a number of elements less the one third of the largest cluster are associated to noise modes and are removed from the stabilization diagram.

\subsection{Post-processing to improve accuracy}

The last phase of the MPE procedure is aimed at improving the accuracy of the mode estimates by applying simple statistical rules. It basically consists of three steps: 1) repeating the clustering procedure using the references obtained by the clustering process in order to improve the estimates accuracy by possibly recovering estimates lost at the beginning of the agglomerative process, 2) recognizing any possible replications of the physical modes in the stabilization diagram, 3) removing the outliers by applying a simple statistical tool based on the box-plot rule.

a)

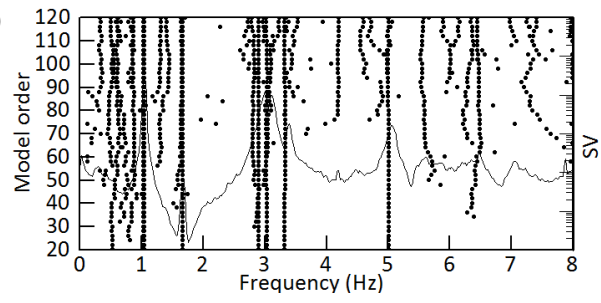

b)

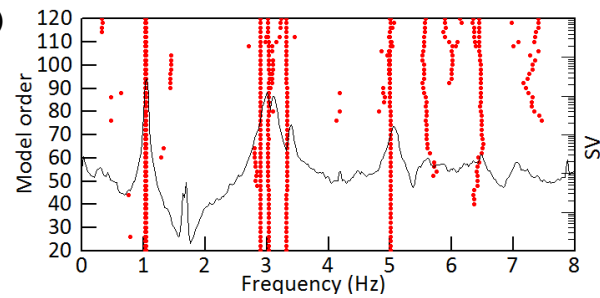

c)

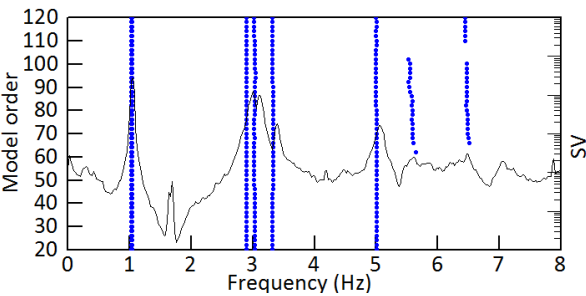

d)

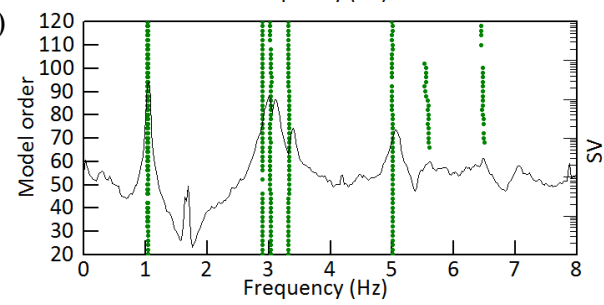

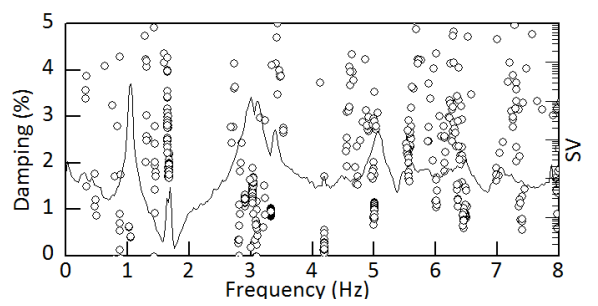
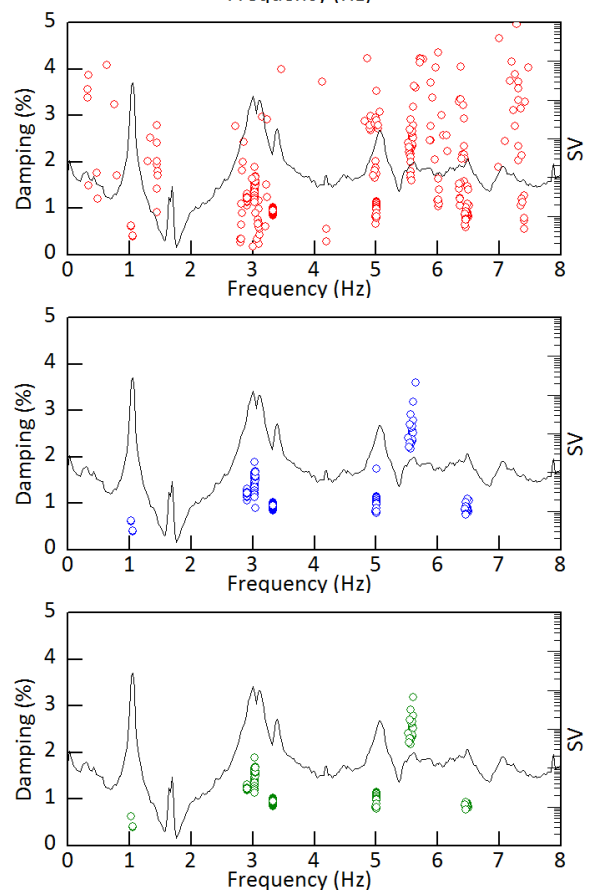

Fig. 1. Stabilization diagrams: (a) after the check on damping ratios; (b) after the check on modal complexity; (c) after clustering; (d) after the post-processing to improve accuracy (final results).

The final outputs of the proposed procedure are the mean values of the modal estimates (mean natural frequency, mean mode shape and median modal damping ratio) belonging to each cluster. The performance of the MPE procedure is exemplified in Fig. 1, by using one dataset collected in the continuous dynamic monitoring of the San Gottardo bell-tower 
(Milan, Italy). In Fig. 1a, the SSI outputs obtained for increasing model's order are plotted. The estimates obtained after the removal of most spurious poles are shown in Fig. $1 \mathrm{~b}$ the resulted stable alignments that stand out after the application of the clustering procedure are shown in Fig. 1c. Finally, Fig. 1d reports the stabilization diagram after the application of the post-processing aimed at improving the accuracy of the results.

\section{Modal tracking}

After the automated MPE, the MT allows to track the evolution in time of natural frequencies and other modal parameters and it is based on the choice of a baseline list of the parameters to be tracked. Although MT represents the very initial step of any OMA-based strategy of SHM, a few strategies have been developed in the literature to accurately manage the MT phase. A common approach adopted to link the currently identified modes to the baseline ones consists of checking the similarity in terms of natural frequencies and mode shapes (through the MAC index). As shown in [3], this approach might fail when the modal parameters exhibit high fluctuation that depends on the variation of environmental and operational conditions: this issue is easily solved by manually adjusting some thresholds but requires an action from the user. A good solution to this issue was proposed in [5] and is based on the automatic definition of adaptive rejection distances, or "distance thresholds", between the current modes and the baseline ones. Nevertheless, even the use of adaptive thresholds might lead to drawbacks when dealing with very simple monitoring systems, because the availability of a limited number of sensors does not always guarantee a good discrimination between different mode shapes. In order to make more robust the procedure introduced in [5], the MT procedure herein presented involves the use of: (a) a static list of reference modes, associated with adaptive rejection thresholds and (b) a dynamic list of reference modes, associated to fixed thresholds. It should be noticed that the automated MT procedure is fully active after an initial training period, in which a minimum number of structural modes are stored to define the initial adaptive thresholds. During this initial period, two a-priori threshold values are assumed to link the current estimates to the baseline. Once the training period has expired, a simple statistical approach is applied to define the adaptive thresholds for each reference mode as shown in the follow equation:

$$
d_{i-r e f, j}^{f}=\sqrt{\operatorname{std}\left(d_{i-r e f, j}^{f}\right)} \quad d_{i-r e f, j}^{M A C}=\sqrt{\operatorname{std}\left(d_{i-r e f, j}^{M A C C}\right)}
$$

where $s t d\left(d_{i, r e f-j}\right)$ and $s t d\left(d^{M A C} C_{i, r f-j}\right)$ are the standard deviation of the distance - computed in terms of natural frequency and MAC, respectively - between the $j$-th estimates $\left(j=1 \ldots n_{\max }\right)$ related to $i$-th reference mode. For present work, the maximum number of values used to define the threshold $\left(n_{\max }\right)$ was equal to 480 because the tracking-window was set at 5 days. It is worth noting that the tracking regions defined by the adaptive thresholds (2) could overlap for closely-spaced modes with relatively similar modal components so that inaccurate associations might arise. To avoid this possibility, the association of the current estimates is positively completed when a further check is satisfied: the candidate mode is compared with the last linked mode, available from the previous tracking, using preselected thresholds. Those thresholds have been set equal to 0.05 for frequency variation and 0.90 for MAC index, respectively.

Following this strategy, the proposed MT procedure complies with three important aspects: 1) accounting for the changes of modal parameters induced by environmental and operational conditions, 2) producing an accurate evolution of the structural modes, even in case of closely space modes with similar mode shapes, and 3) conceivably reducing the number of outliers during the tracking process. 


\section{Modal identification of the San Gottardo bell-tower}

The proposed methodology is currently used to automatically manage the continuous dynamic monitoring of the bell-tower of the church of San Gottardo in Corte in Milan, Italy. The bell-tower, completed in 1336, is about $54.0 \mathrm{~m}$ high and consists of a stone masonry square basement of $12.0 \mathrm{~m}$, an octagonal portion in solid brick masonry until the height of $41.0 \mathrm{~m}$ and a high cusp. Following the current trend [6, 7] adopted in SHM of historic towers, a simple dynamic monitoring system (consisting of 2 bi-axial seismometers, 24-bit digitizer and UMTS modem for data transfer) was installed in the bell chamber of the tower at the beginning of November 2016.

Fig. 1 shows the performance of the MPE procedure applied to a 1-hour dataset recorded on $21 / 03 / 2017$. The figure exemplifies the typical cleaning action exerted by the consecutive steps of the MPE procedure on the stabilization diagram (as well as on the frequency vs damping representation). The plots in Fig. 1d contain the final results, i.e. the alignments of stable poles corresponding to the identified vibration modes. It should be noted that: i) within the investigated frequency range $(0-8 \mathrm{~Hz}), 8$ vibration modes were successfully identified, with 6 of these modes (alignments of stable poles) corresponding to amplification regions of the first SV line; (b) the last two modes, although weakly excited, are clearly identified when the model order increases; (c) as it is common for historic towers (see e.g. [6,7]), the natural frequencies of the two lower modes are closely spaced. Fig. 2 shows the evolution in time of the natural frequencies of all modes previously identified (Fig. 1). Furthermore, frequency fluctuations are clearly detected and conceivably associated with the changing environmental conditions (i.e., temperature and humidity).

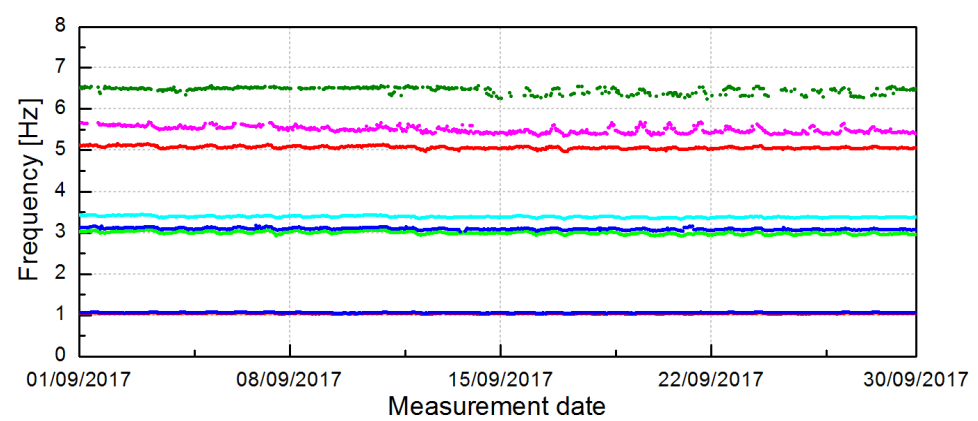

Fig. 2. San Gottardo bell-tower: time evolution (September 2017) of the natural frequencies.
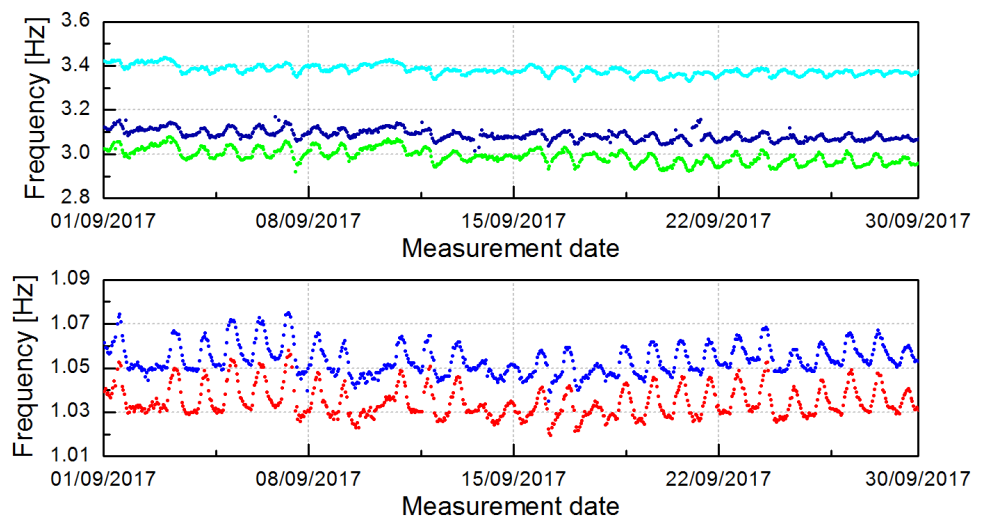

Fig. 3. San Gottardo bell-tower: environmental effects on the identified natural frequencies. 
Fig. 3 shows zooms of the natural frequencies of the two lower modes to better demonstrate the effects of environmental conditions.

It is worth mentioning that, during the tower monitoring, the mode shape associated with the third frequency (around $3 \mathrm{~Hz}$ ) exhibits important changes. This unlikely condition and the availability of only 4 channels of data make the automated tracking difficult. In fact, the mode shape variations result in high value of the adaptive thresholds (2) and the thresholds associated to closely-spaced modes tend to produce an overlapping region.

As previously pointed out, to avoid possible incorrect associations, the linking process is completed with a further validation between the current mode with the last one identified (playing the role of dynamic reference), that progressively change as the MT process runs. Following this strategy, a more robust association between reference static mode and candidate mode is obtained, producing an accurate evolution of the structural modes and reducing the number of outliers and incorrect associations during the tracking process even in case of closely space mode with similar mode shapes.

\section{Conclusions}

An automated modal identification procedure, based on parametric methods and on the automatic interpretation of stabilization diagrams, has been presented. The performance of the proposed algorithm has been demonstrated using real data collected during permanent dynamic monitoring of a historic masonry bell tower.

The main contributions of the paper can be summarized as follow: (i) the pre-filtering check, based on damping and complexity modes checks, has proven quite effective in cleaning the results obtained by the SSI method, allowing the elimination of most of the spurious modes, (ii) the application of simple statistical tools in the post-processing phase permits the reduction of uncertainty related to the results, (iii) the double check carried out during the tracking process turns out to provide a robust association between candidate modes and reference mode providing a fairly good identification rate even in case of closely spaced modes and weakly excited modes.

Moreover, the developed procedure is particularly suitable for continuous dynamic monitoring of structure that involves the use of stabilization diagrams. Furthermore, the robust performance of the permanent monitoring process is the base-step for further damage detection procedure based on the use the modal parameters.

\section{References}

1. B. Peeters, G. De Roeck, Mech. Syst. Signal Pr. 13, 855-878, (1999)

2. G. Marrongelli, F. Magalhães, Á. Cunha, Procedia Engineering 199, 2172-2177, (2017)

3. F. Magalhães, Cunha, E. Caetano, Mech. Syst. Signal Pr. 23, 316-329, (2009)

4. E. Reynders, J. Houbrechts, G. De Roeck, Mech. Syst. Signal Pr. 29, 228-250, (2012)

5. A. Cabboi, F. Magalhães, C. Gentile, Á. Cunha, Struct. Control Hlth. 24(1), e1854, (2017)

6. F. Ubertini, G. Comanducci, N. Cavalagli, Struct. Hlth. Monit. 15, 438-457, (2016)

7. C. Gentile, M. Guidobaldi, A. Saisi, Meccanica 51, 2873-2889, (2016) 